\title{
Capacity and Delay Tradeoffs in Mobile Networks Under Gaussian Channel Model
}

\author{
Cheng Wang*, Xiang-Yang $\mathrm{Li}^{* \dagger}$, Shaojie Tang ${ }^{\dagger}$, Changjun Jiang* \\ * Department of Computer Science, Tongji University, Shanghai, China \\ $\dagger$ Department of Computer Science, Illinois Institute of Technology, Chicago, IL, 60616
}

\begin{abstract}
Extensive efforts have been made to study the asymptotic capacity, delay, and their tradeoffs for large-scale mobile ad hoc networks, under different mobility models and communication models. Majority results adopt the fixed-rate communication model, such as the protocol model and physical model, and none of them breaks the limitation of tradeoffs: delay $/$ capacity $=\omega(1)$ so far, even for the simplest i.i.d. model. In this work, we investigate this problem under the Gaussian channel model, and demonstrate that the delay-capacity tradeoffs can be further improved by designing new two-hop strategy under a general mobility model, called hybrid random walk mobility model (HRWMM). We found that the capacity and delay have several regions, depending on the freedom degree $\gamma \in[0,1]$ of mobile nodes. Specifically, we show that under the prerequisite of ensuring the optimal per-session capacity, i.e., of order $\Theta(1)$ : (1) for $0<\gamma \leq 1$, the optimal delay under the Gaussian channel model is smaller than that under the protocol model or physical model; (2) for $\gamma=0$, i.e., ordinary random walk model, it is not larger than the delay under the protocol model or physical model; (3) for $\gamma=1$, i.e., i.i.d. model, the capacity and delay can simultaneously achieve the optimal order, i.e., $\Theta(1)$.
\end{abstract}

\section{INTRODUCTION}

In mobile ad hoc networks (MANETs), the mobility of nodes brings challenges in the performance analysis of network due to the frequent change of topology. A milestone work by Grossglauser and Tse [1] showed that the MANET is scalable in terms of unicast capacity with assistance of the mobility, while the static ad hoc networks are not scalable [2], [3]. However, such a significant gain in capacity is obtained at the cost of a very large delay. Since the capacity and delay are both important in some application scenarios of MANETs, it is necessary to examine the relationship between them, i.e., the delay-capacity tradeoffs [4]. The delay and capacity in MANETs depend on the nature of the mobility model. The mobility models that have been studied include i.i.d. mobility model [4]-[7], random walk mobility model [8], Brownian mobility model [8]-[10], random way-point mobility model [9], [11], [12], and other mobility models [13]-[17].

In the literature, most of works adopt the fixed-rate communication model under which if the value of a given conditional expression is beyond the threshold, the transmitter can send the data successfully to the receiver at a specific constant rate; otherwise, it cannot send data at any rate. The protocol model and physical model defined in [2] both belong to fixed-rate communication models. Under these models, a uniform lower bound on the delay-capacity tradeoffs for any common mobility models is that delay/capacity $=\omega(1)$, which implies that the optimal capacity must be achieved at the cost of a very large (infinite) delay, even for the simplest i.i.d. model, [18]-[22].

Besides the fixed-rate model, the wireless transmission is often characterized as a model that determines the transmission rate at which the transmitter can send its data to the receiver reliably, based on a continuous function of the receiver's SINR. In this work, we call this type of models adaptiverate communication models. The Gaussian channel model [3], [23], [24] belongs to this category. Unlike the fixed-rate model, the adaptive-rate model permits the adaption of link rate according to the condition of channels. A question is whether the limitation of delay-capacity tradeoffs under the fixed-rate communication model can be broken by adopting the adaptive-rate communication model, such as Gaussian channel model. We aim to study this question in this work, and our results provide a positive answer.

Here we introduce a hybrid random walk mobility model (HRWMM) [12], [25], denoted by $\mathbb{M}(\gamma)$, that is built by slightly modifying the hybrid random walk model proposed in [12] (see Section II-A for details). Here, the constant $\gamma \in[0,1]$ is a parameter denoting the freedom degree (freedom exponent) of mobile nodes. As $\gamma$ goes to 1 , the freedom degree is increasing; when $\gamma=1$, the freedom degree is extremely large, and the mobility model is essentially the i.i.d. mobility model. On the other hand, when $\gamma=0$, the freedom degree of nodes under HRWMM is extremely limited, and it essentially becomes the ordinary random walk model, [12], [25].

We adopt a modified version of the well-known two-hop strategy [1] that has been extensively used under the protocol model or physical model. Our scheme is still a simple threshold-based method: when the distance between two nodes is at most of a threshold $\mathfrak{l}_{\mathbf{S}}$, these two nodes are requested to communicate directly; otherwise, they will communicate via the two-hop relay strategy. To some extent, this work focuses more on analyzing the asymptotic behavior under the Gaussian channel model and the HRWMM mobility model rather than designing a new communication strategy.

Main Contributions: Our major contributions of this work are as follows:

- To the best of our knowledge, this is the first work to study the asymptotic capacity and delay bounds for MANETs under the Gaussian channel model, instead of the protocol model or physical model. 
- Depending on the critical parameter $\mathfrak{l}_{\mathbf{S}}:[1, \sqrt{n}]^{1}$ of a given two-hop strategy $\mathbf{S}$ under the Gaussian channel model $\mathscr{C}_{\text {gau }}(\alpha)$ with a power attenuation exponent $\alpha>2$, we derive the asymptotic per-session capacity $\lambda(\mathbf{S}, n)$ and average delay bounds $\mathbf{E}(D(\mathbf{S}, n))$ under the hybrid random walk model $\mathbb{M}(\gamma)$ as following:

(a) when $\gamma=0$,

$$
\begin{gathered}
\lambda(\mathbf{S}, n)=\left\{\begin{array}{lll}
\Theta(1) & \text { when } & \mathfrak{l}_{\mathbf{S}}:[1, \sqrt{\log n}] \\
\Omega\left(\frac{\log n}{\left(\mathfrak{l}_{\mathbf{S}}\right)^{2}}\right) & \text { when } & \mathfrak{l}_{\mathbf{S}}:(\sqrt{\log n}, \sqrt{n}]
\end{array}\right. \\
\mathbf{E}(D(\mathbf{S}, n))=\left\{\begin{array}{lll}
\Theta(n \log n) & \text { when } & \mathfrak{l}_{\mathbf{S}}=\Theta(1) \\
\Omega\left(\frac{n}{\log n}\right) & \text { when } & \mathfrak{l}_{\mathbf{S}}:(1, \sqrt{n}) \\
O\left(\frac{n}{\log n}\right) & \text { when } & \mathfrak{l}_{\mathbf{S}}=\Theta(\sqrt{n})
\end{array}\right.
\end{gathered}
$$

Please see the illustration in Fig.1(a).

(b) when $0<\gamma<1$,

$$
\begin{aligned}
& \lambda(\mathbf{S}, n)=\left\{\begin{array}{lll}
\Theta(1) & \text { when } & \mathfrak{l}_{\mathbf{S}}:\left[1, \sqrt{n^{\gamma} \log n}\right] \\
\Omega\left(\frac{\log n \cdot n^{\gamma}}{\left(\mathfrak{l}_{\mathbf{S}}\right)^{2}}\right) & \text { when } & \mathfrak{l}_{\mathbf{S}}:\left(\sqrt{n^{\gamma} \log n}, \sqrt{n}\right]
\end{array}\right. \\
& \mathbf{E}(D(\mathbf{S}, n))=\left\{\begin{array}{lll}
\Theta\left(\frac{n}{\left(l_{\mathbf{S}}^{2}\right.}\right) & \text { when } & \mathfrak{l}_{\mathbf{S}}:\left[1, \sqrt{\frac{n^{\gamma}}{\log n}}\right] \\
\Theta\left(\frac{\log n}{n^{\gamma-1}}\right) & \text { when } & \mathfrak{l}_{\mathbf{S}}:\left[\sqrt{\frac{n^{\gamma}}{\log n}}, n^{\frac{\gamma}{2}}\right] \\
\Omega\left(\frac{n^{1-\gamma}}{\log n}\right) & \text { when } & \mathfrak{l}_{\mathbf{S}}:\left(n^{\frac{\gamma}{2}}, \sqrt{n}\right) \\
O\left(\frac{n^{1-\gamma}}{\log n}\right) & \text { when } & \mathfrak{l}_{\mathbf{S}}=\Theta(\sqrt{n})
\end{array}\right.
\end{aligned}
$$

Please see the illustration in Fig.1(b).

(c) when $\gamma=1, \lambda(\mathbf{S}, n)=\Theta(1)$,

$\mathbf{E}(D(\mathbf{S}, n))=\left\{\begin{array}{lll}\Theta\left(\frac{n}{\left(l_{\mathbf{S}}\right)^{2}}\right) & \text { when } & \mathfrak{l}_{\mathbf{S}}:\left[1, \sqrt{\frac{n}{\log n}}\right) \\ \Theta(\log n) & \text { when } & \mathfrak{l}_{\mathbf{S}}:\left[\sqrt{\frac{n}{\log n}}, \sqrt{n}\right) \\ \Theta(1) & \text { when } & \mathfrak{l}_{\mathbf{S}}=\Theta(\sqrt{n})\end{array}\right.$

Please see the illustration in Fig.1(c).

- Based on the results described in Fig.1, we can extract some interesting results as following:

(a) Under the classical two-hop strategy, to achieve the per-session capacity of optimal order, i.e., $\Theta(1)$, the critical distance is set to be $\mathfrak{l}_{\mathbf{S}}=\Theta\left(\frac{1}{\sqrt{n}}\right)$ under the protocol model or physical model [1]. Then, it is intuitive that by a simple geometric scaling, i.e., by letting $\mathfrak{l}_{\mathbf{S}}=\Theta(1)$, the capacity for extended networks can be achieved of a constant order. We first prove that the constant-order capacity is achievable under the Gaussian channel model as long as $\mathfrak{l}_{\mathbf{S}}:\left[1, \min \left\{n^{\frac{\gamma}{2}} \sqrt{\log n}, \sqrt{n}\right\}\right]$.

(b) Under the prerequisite of ensuring the optimal persession capacity, i.e., of order $\Theta(1)$, when $0<\gamma \leq 1$, the optimal delay under the Gaussian channel model (GCM) is smaller than that under the protocol model (ProM) or physical model (PhyM); and when $\gamma=0$ (random walk model), it is not larger than the delay under ProM or PhyM. Fig.2 shows the comparison between the delay for extended networks under

\footnotetext{
${ }^{1}$ For conciseness, we let the expression $f(n):\left[\phi_{1}(n), \phi_{2}(n)\right]$ represent that $f(n)=\Omega\left(\phi_{1}(n)\right)$ and $f(n)=O\left(\phi_{2}(n)\right)$; let $f(n):\left(\phi_{1}(n), \phi_{2}(n)\right)$ represent that $f(n)=\omega\left(\phi_{1}(n)\right)$ and $f(n)=o\left(\phi_{2}(n)\right)$.
}
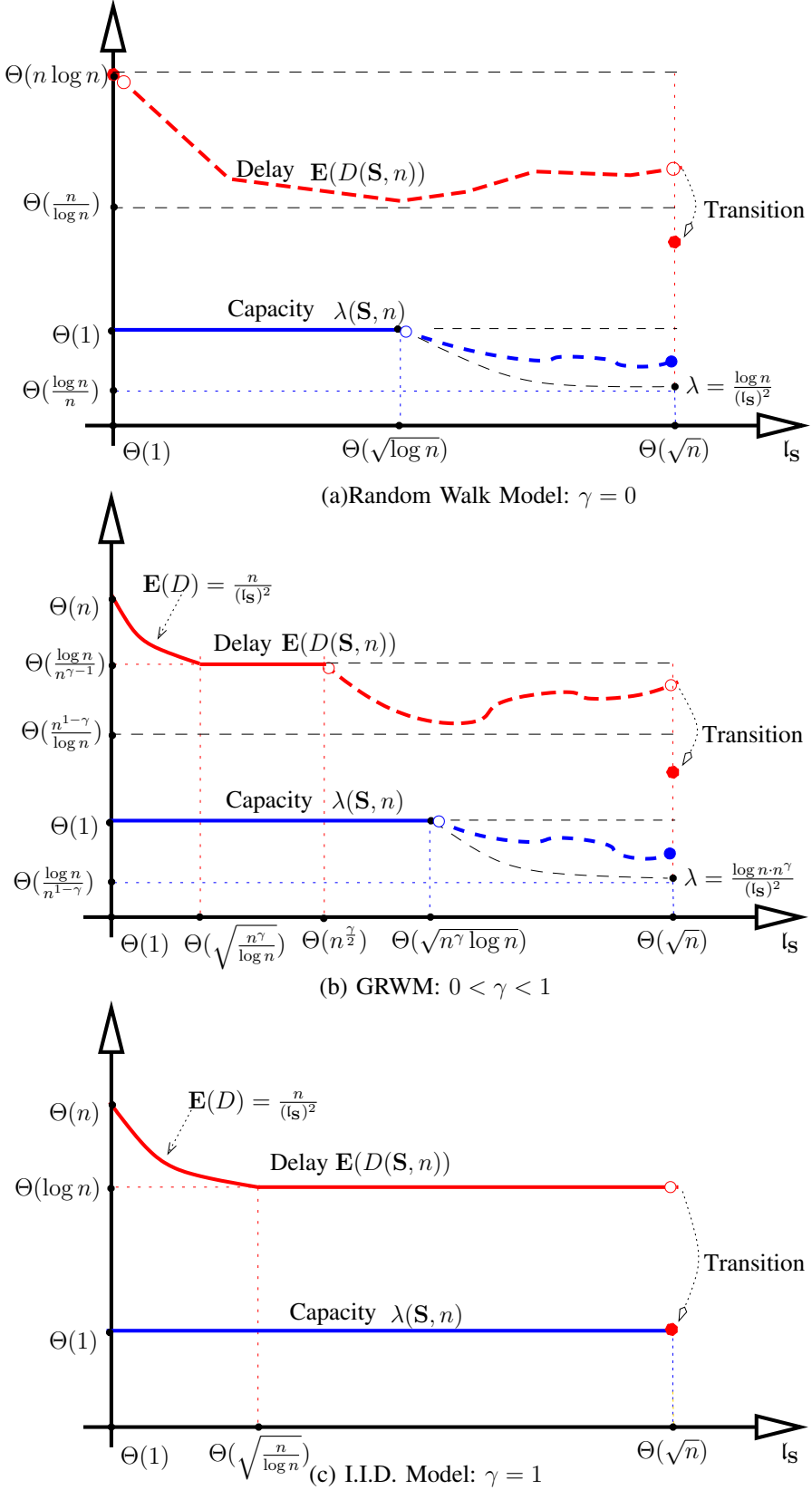

Fig. 1. Capacity and delay depending on the freedom degree $\gamma \in[0,1]$. The solid curves denote the functions of delay and capacity in terms of $\mathfrak{l}_{\mathbf{S}}$. The dashed curves denote the bounds on the functions of delay and capacity rather than the precise functions.

GCM and that under ProM (or PhyM), when the capacity of optimal order is achievable.

(c) For any $\gamma \in[0,1]$, when the capacity is sustained of order $\Theta(1)$, the delay is not increasing with $\mathfrak{l}_{\mathbf{S}}$; when $\mathfrak{l}_{\mathbf{S}}=\Theta(\sqrt{n})$, a transition of delay occurs. For $\gamma=1$, i.e., i.i.d. model, there is a transition of the optimal delay: the capacity and delay can simultaneously achieve the optimal order, i.e., $\Theta(1)$, by the simple two-hop strategy. Please see the illustrations in Fig.1(c) and Fig.3. This ideal result comes from the extreme high freedom $(\gamma=1)$ of nodes under i.i.d. mobility model and the advantage of adaptive rate 


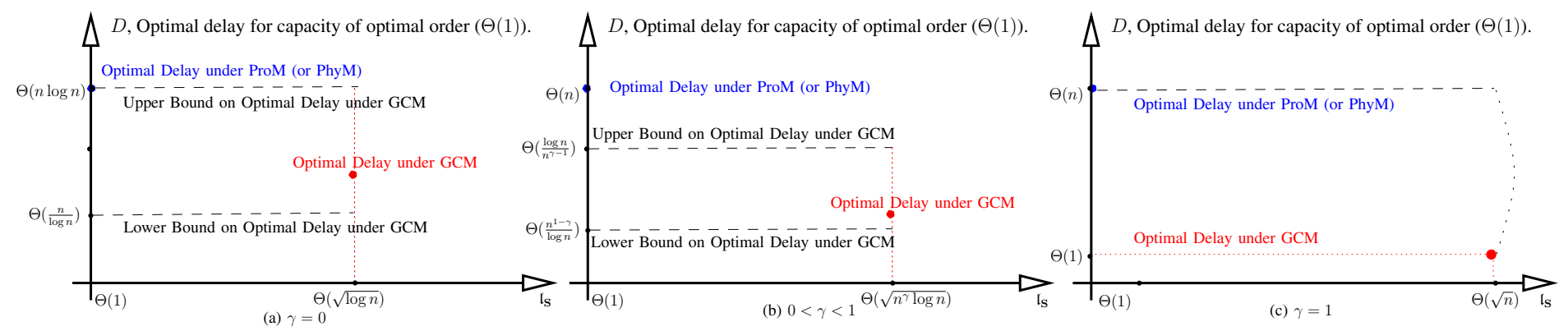

Fig. 2. Optimal delay for the optimal capacity (of order $\Theta(1)$ ) and the corresponding critical parameter $\mathfrak{l}_{\mathbf{S}}$ under the protocol model (ProM) or physical model (PhyM) and Gaussian channel model (GCM).

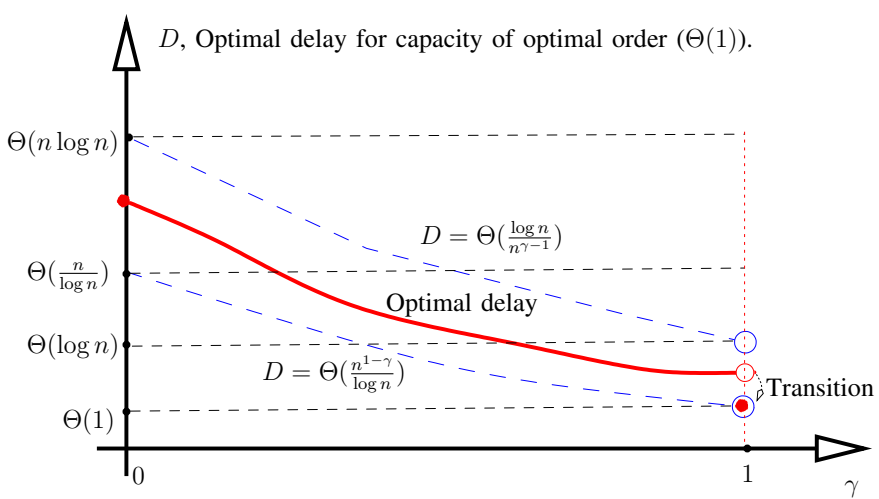

Fig. 3. Optimal delay depending on the freedom degree $\gamma$. The solid curve (including the single node) is the illustration of optimal delay in terms of $\gamma$; the dashed curves denote the upper and lower bounds of the optimal delay.

under the Gaussian channel model. Indeed, this result breaks the limitations of capacity-delay trade-offs derived under the protocol model or physical model in the literature, [13], [14], [17]-[19], [26]. We state that the transition is the result of the singularity of i.i.d. model for extended networks.

(d) The capacity is independent of the specific value of power attenuation exponent of Gaussian channel model if $\alpha>2$, although it decreases with $\mathfrak{l}_{\mathbf{S}}$ under some regimes. The reason for this phenomenon lies in the fact that as the link rate changes with the link length under Gaussian channel model, the data transmitted via long-distance links are indeed infinitesimal relative to those via short links when $\alpha>2$. The derivative process can be found in Theorem 1 .

The rest of the paper is organized as follows. We formulate the system model in Section II. In Section III, we introduce the classical two-hop strategy, and propose some properties of extended mobile ad hoc networks, which will be used in the analysis of delay and capacity done in Section IV. Finally, we draw some conclusions and future perspective in Section V.

\section{A. Network Model}

\section{SySTEM MODEL}

We consider an ad hoc network consisting of $n$ mobile nodes, distributed uniformly on a square region $\mathcal{R}(n)=$ $[0, \sqrt{n}] \times[0, \sqrt{n}]$ initially. After that, all mobile nodes move in
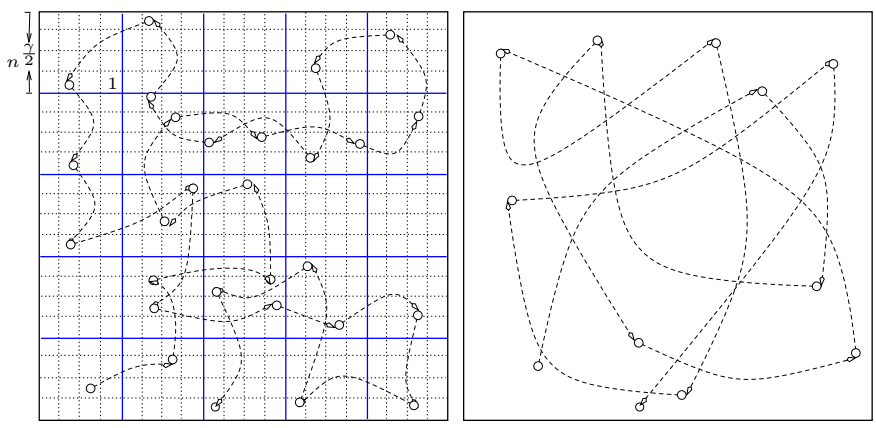

Fig. 4. The motion of a node under the mobility model. The model is defined based on the lattices $\mathbb{L}(n, 1)$ and $\mathbb{L}\left(n, n^{\gamma}\right)$. As illustrated in the right figure, when $\gamma=1$, the model is specialized to an i.i.d. mobility model (I.I.D. MM).

accordance with the hybrid random walk model introduced in Section II-B. It is possible for a source node to send packets to its destination node via multiple relays.

\section{B. Mobility Model}

We introduce a hybrid random walk mobility model (HRWMM) by slightly modifying the hybrid random walk model proposed in [12], [25]. Partition a square of area $\mathfrak{a}$ into $\frac{\mathfrak{a}}{\mathfrak{c}}$ square cells of area $\mathfrak{c}$, let $\mathbb{L}(\mathfrak{a}, \mathfrak{c})$ denote the resulted lattice for ease of presentation. To avoid some trivialities, we assume that $\sqrt{\frac{\mathfrak{a}}{\mathfrak{c}}}$ is always an integer. Divide the deployment region $\mathcal{R}(n)=[0, \sqrt{n}]^{2}$ into $n$ squares of area 1 (henceforth referred as cells), resulting a lattice $\mathbb{L}(n, 1)$. We next divide the region $\mathcal{R}(n)$ into $n^{1-\gamma}$ squares of area $n^{\gamma}$ (henceforth referred as super cells) for $\gamma \in[0,1]$, resulting a lattice $\mathbb{L}\left(n, n^{\gamma}\right)$. Clearly, there are $n^{\gamma}$ cells in each super cell. Each cell (or super cell) is assigned a 2-D index $(x, y)$ if it is in the $(x+1)$ th column and $(y+1)$ th row of lattice $\mathbb{L}(n, 1)$ (or of lattice $\mathbb{L}\left(n, n^{\gamma}\right)$ ), in the order from left to right and from bottom to top, e.g., the cell in bottom left corner is indicated as $(0,0)$. To deal with the edge effects [2], we treat the deployment region $\mathcal{R}(n)$ as a 2-D torus. The cells (or super cells) adjacent to cell (or super cell) $(i, j)$ are the cells (or super cells) $(i+1, j),(i-1, j)$, $(i, j+1)$, and $(i, j-1)$, where the addition and subtraction operations are performed modulo $n$ (or $n^{1-\gamma}$ ).

Time is divided into phases of equal unit duration. Without loss of generality, we assume the duration of each phase is $L_{\mathrm{p}}=1$. Initially, each node is equally likely in any of the 


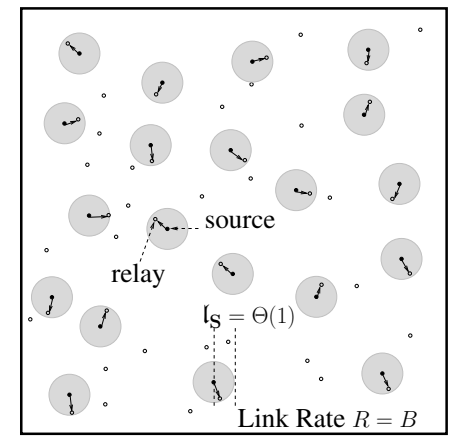

(a) Phase 1 in Strategy under F-R Model

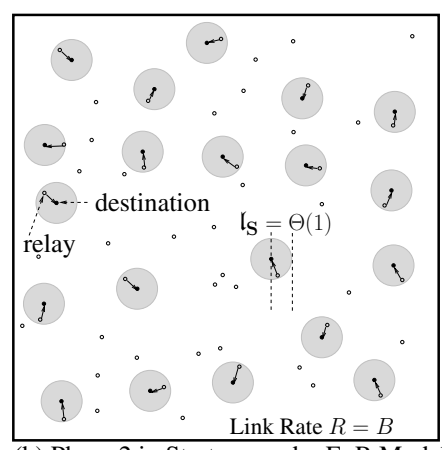

Link Rate $R=B$

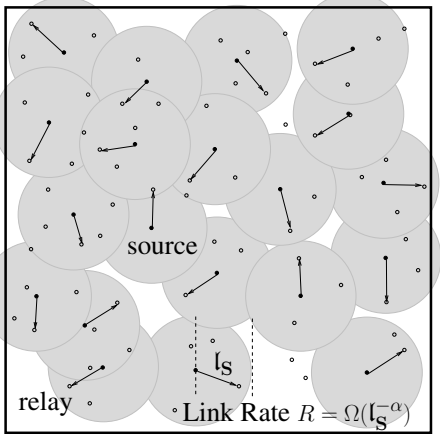

(c) Phase 1 in Strategy under GCM

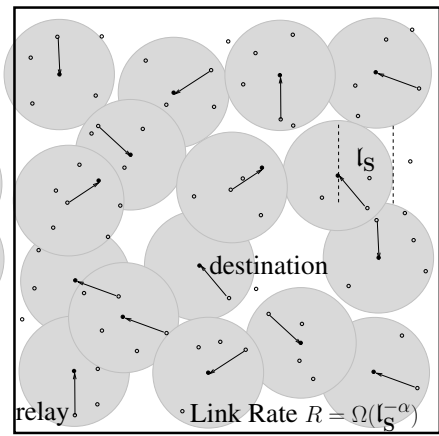

(d) Phase 2 in Strategy under GCM

Fig. 5. Under a given strategy $\mathbf{S}$, a key parameter is the critical distance $\mathfrak{l}_{\mathbf{S}}:[1, \sqrt{n}]$, within which two nodes will communicate directly. Under the fixed-rate model (F-R model, Figures (a) and (b)), e.g., the protocol model and physical model, the only feasible setting of $l_{\mathbf{S}}$ for achieving the capacity of $\Theta(1)$ for random extended networks is of $\mathfrak{l}_{\mathbf{S}}=\Theta(1)$, which intuitively causes large delays. During each time slot, there are $\Theta(n)$ feasible links (sender-receiver pairs) of length $O(1)$ along which a fixed constant rate can be sustained. Under Gaussian channel model (GCM, Figures (c) and (d)), the link rate is adjustable according to SINR at the receiver. It is possible to achieve a constant capacity with $\mathfrak{l}_{\mathbf{S}}=\omega(1)$, which possibly decreases network delay. In each slot, $\Theta(n)$ links of length $O\left(\mathfrak{l}_{\mathbf{S}}\right)$ can simultaneously achieve the rate of order $\Omega\left(\mathfrak{l}_{\mathbf{S}}^{-\alpha}\right)$, where $\alpha>2$ is the power attenuation exponent.

cells, independent of the other nodes. At the beginning of each phase, a node uniformly chooses one cell at random from a randomly selected adjacent super cell, and jumps to the new cell from its current cell. And it will stay at the new cell during this phase. Please see the illustration in Fig. 4. Finally, as $\gamma \rightarrow$ 1 , the freedom degree of the mobile nodes increases. When $\gamma=1$, the mobility model is essentially the i.i.d. mobility model [12], [25].

\section{Communication Model}

In this section, we aim to select the optimal communication model from some classical models.

When time is divided into slots of sufficiently small duration, it is reasonable to assume that the position of each node is invariable (approximately) during a slot. Then, how small duration, denoted by $L_{\mathrm{s}}$, can ensure the above assumption? Since the motion of every node happens instantaneously at the beginning of each phase, it follows that the position of each node remains the same during a whole phase. Hence, we can set $L_{\mathrm{s}}=L_{\mathrm{p}}=1$. We call the time slots of duration $L_{s}$ static slots in the following content. Intuitively, we can treat the MANET as a static network during a static slot. Note that based on $n$ mobile nodes, there are $n(n-1)$ possible directed communication links each of which is associated with a unique pair of transmitter and receiver. For any directed link $i$, we use $\mathbf{t}_{i}$ and $\mathbf{r}_{i}$ to denote its transmitter and receiver. Different from a static network, the position of each node may vary significantly as time goes on. For ease of explanation, let $i^{t}$, $\mathbf{t}_{i}^{t}$ and $\mathbf{r}_{i}^{t}$, denote $i, \mathbf{t}_{i}$ and $\mathbf{r}_{i}$ presented in the static slot $t$, respectively.

Generally, there are three classical types of networkingtheoretic communication models in the study of scaling laws of wireless networks: the protocol model [2], physical model [2] and Gaussian channel model [3], [24]. We only introduce the latest one, while the detailed definitions of the protocol model and physical model are moved into Appendix A-A of our technical report [27] due to limited space. Assume that the bandwidth is $B=\Theta(1)$, and let $|\cdot|$ denote the
Euclidean distance between two nodes. Let $\mathscr{C}_{\text {gau }}(\alpha)$ denote the family consisting of all sets of available links that can be simultaneously scheduled under the Gaussian channel model.

Definition 1: Under Gaussian channel model $\mathscr{C}_{\text {gau }}(\alpha)$, for any scheduled set of links, say $\mathcal{S}^{t}$, the rate of a link, say $i^{t}$, is achieved of

$$
R_{i}^{\text {gau }, t}=B \times \mathbf{1} \cdot\left\{i^{t} \in \mathcal{S}^{t}\right\} \times \log \left(1+\operatorname{SINR}_{i}^{t}\right),
$$

where $\operatorname{SINR}_{i}^{t}=\frac{P \cdot \ell\left(\mathbf{t}_{i}^{t}, \mathbf{r}_{i}^{t}\right)}{N_{0}+\sum_{j \in \mathcal{S}^{t}-\{i\}} P \cdot \ell\left(\mathbf{t}_{j}^{t}, \mathbf{r}_{i}^{t}\right)}$, and $\ell(\cdot)$ is the power attenuation function.

The wireless propagation channel typically includes path loss with distance, shadowing and fading effects. As in [2], [3], [24], we assume that the channel gain depends only on the distance between the transmitter and receiver, and ignore shadowing and fading. Following the setting in [3], [28], for extended networks studied here, we let $\ell\left(\mathbf{t}_{i}^{t}, \mathbf{r}_{i}^{t}\right)=$ $\min \left\{1,\left|\mathbf{t}_{i}^{t}-\mathbf{r}_{i}^{t}\right|^{-\alpha}\right\}$, where $\alpha>2$ is the power attenuation exponent.

Taking both the practical reality and capacity optimization into consideration, we state that Gaussian channel model is the optimal communication model among these three models. Please see the detailed analysis in Appendix A-C of our technical report [27].

\section{Capacity and Delay}

We introduce the definitions of capacity [1], [12], [29] and delay [12], [29] in mobile ad hoc networks.

1) Network Capacity: The definition of network capacity is based on the stability of network. Data packets are assumed to arrive at node $v_{i}$ with probability $\lambda_{i}$ during each slot, i.e., in a Bernoulli process of arrival rate $\lambda_{i}$. The network is stable if there exists a scheduling scheme under which the queue at each node does not expand to infinity as time goes to infinity. Thus, the per-session capacity of the network is the maximum rate that the network can stably support.

2) Network Delay: The delay for a packet is defined as the time it takes the packet to reach its destination after it arrives 


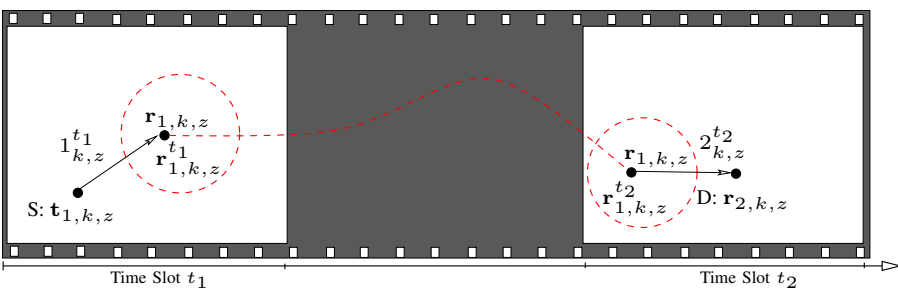

Fig. 6. Illustration of the routing path of Packet $z$ for session $k, \mathcal{P}_{k, z}=$ $\left\{1_{k, z}^{t_{1}}, 2_{k, z}^{t_{2}}\right\} . \mathbf{t}_{1, k, z}$ is the source node, $\mathbf{r}_{2, k, z}$ is the destination node; $\mathbf{r}_{1, k, z}$ is the receiver of link $1_{k, z}^{t_{1}}$ and the transmitter of link $2_{k, z}^{t_{2}} \cdot \mathbf{r}_{1, k, z}^{t_{1}}$ and $\mathbf{r}_{1, k, z}^{t_{2}}$ denote the positions of node $\mathbf{r}_{1, k, z}$ at the static slot $t_{1}$ and $t_{2}$, respectively.

at the source. The total network delay is the expectation of the average delay over all packets in the long term.

\section{Communication Strategy}

Under a given communication strategy $\mathbf{S}$, a key parameter is the critical distance, denoted by $\mathfrak{l}_{\mathbf{S}}$, within which two nodes can communicate directly. Then, it holds that $\mathfrak{l}_{\mathbf{S}}:[1, \sqrt{n}]$. Please see the illustrations in Fig.5. Depending on a specific mobility model and the critical distance $\mathfrak{l}_{\mathbf{S}}$, we can define the contact interval [11] during which data can be transmitted continually between the nodes with a distance of order $O\left(\mathfrak{l}_{\mathbf{S}}\right)$; and we define the waiting interval as the time that it takes a packet at a relay node to wait for the next transmission. We design the communication strategy based on the lattice $\mathbb{L}(\mathfrak{a}, \mathfrak{c})$ defined in Section II-B. Then, under the strategy $\mathbf{S}$, we always say that two nodes can communicate directly if they are located in the same cell in $\mathbb{L}\left(n,\left(\mathfrak{l}_{\mathbf{S}}\right)^{2}\right)$.

Since this is the first work, to the best of our knowledge, studying the asymptotic capacity and delay under the Gaussian channel model, we limit the communication strategies to the class without using the replication policy ${ }^{2}$ [5] to stress the new insight different from that under the fixed-rate model, i.e., protocol model or physical model.

\section{A. Classical Two-Hop Strategy}

Two-hop strategy was first proposed by Grossglauser and Tse [1]. Under the two-hop strategy, for each packet $z$ from session $k$, the complete relay path consists of at most two links. We denote such a path by $\mathcal{P}_{k, z}=\left\{1_{k, z}^{t_{1}}, 2_{k, z}^{t_{2}}\right\}$, where $t_{1}$ and $t_{2}$ are two disjointed slots. Note that there is an alternative case in which the packet $z$ is directly transmitted from the source to its destination. For this case, the relay path can be denoted by $\mathcal{P}_{k, z}=\left\{1_{k, z}^{t_{1}}\right\}$.

There are generally three phases under the two-hop strategy: (1) $S \rightarrow R$ phase, during which the source node $\mathbf{t}_{1, k, z}$ transmits the packet $z$ to a relay node $\mathbf{r}_{1, k, z}$, i.e., $\mathbf{t}_{2, k, z}$; (2) waiting phase, during which $\mathbf{r}_{1, k, z}$ holds the packet $z$ until it meets the destination node $\mathbf{r}_{2, k, z}$ within a distance of $\mathfrak{l}_{\mathbf{S}}$, and (3) $R \rightarrow D$ phase, during which $\mathbf{r}_{1, k, z}$ transmits the packet $z$ to $\mathbf{r}_{2, k, z}$. Please see the illustration in Fig.6.

\footnotetext{
${ }^{2}$ Under the replication policy, the source node can send duplicate copies of the packet to new relay nodes.
}

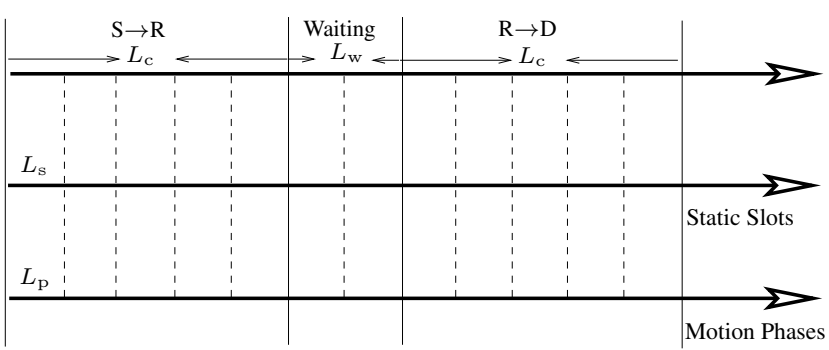

Fig. 7. Decomposition of Two-Hop Communication. $L_{\mathrm{c}}$ and $L_{\mathrm{w}}$ denote the average duration of the contact intervals and waiting intervals, respectively. $L_{\mathrm{S}}$ denotes the duration of static slots. $L_{\mathrm{p}}=1$ denotes the duration of phases under the HRWMM.

Here, the duration of $\mathrm{S} \rightarrow \mathrm{R}$ phase and $\mathrm{R} \rightarrow \mathrm{D}$ phase is of the same order as the contact time with the parameter $l_{S}$ (Definition 4); the duration of the waiting phase can be derived based on the first hitting time (Definition 2, Corollary 1). $\mathrm{S} \rightarrow \mathrm{R}$ phase and $\mathrm{R} \rightarrow \mathrm{D}$ phase are the contact intervals, and the waiting phase is the waiting interval. Please see the illustration in Fig.7. We determine these intervals in Section III-B2 and Section III-B3.

\section{B. Some Properties of Mobile Networks}

The following network properties to be examined depend on the value of $\mathfrak{l}_{\mathbf{S}}$, which denotes the critical distance of a given communication strategy $\mathbf{S}$.

1) Spatial Multiplexing in Extended MANET: Note that if we can design a communication strategy under which $\mathfrak{l}_{\mathbf{S}}=$ $O(1)$, the physical model or protocol model can work as well as Gaussian channel model in terms of reality, [24]. However, in order to ensure the connectivity, we cannot set $\mathfrak{l}_{\mathbf{S}}=O(1)$ under the static random extended network where $n$ nodes are uniformly at random in the region $[0, \sqrt{n}]^{2}$. In contrast, the mobility of nodes in MANETs makes it possible to design such strategy. On the other hand, since we adopt the Gaussian channel model, we can set the parameter $\mathfrak{l}_{\mathbf{S}}$ of our scheme to be any value within $[1, \sqrt{n}]$. Intuitively, there is a trade-off between the throughput and delay according to $\mathfrak{l}_{\mathbf{S}}$.

We carry out our analysis based on lattice $\mathbb{L}(n, \mathfrak{c}(n))$, where $\mathfrak{c}(n)>2$. Denote the cell in the $i+1$ th row and $j+1$ th column by $C_{i, j}$, and denote the number of mobile nodes in $C_{i, j}$ as $n_{i, j}$. Furthermore, we define a sequence of sets of cells. For $h=0,1, \cdots, \mu, v=0,1, \cdots, \mu$, and $0 \leq i, j \leq \sqrt{n / \mathfrak{c}(n)}-1$, we define a set of cells as

$$
\mathcal{C}_{h, v}(\mu):=\left\{C_{i, j} \mid i \bmod (\mu+1)=h, j \bmod (\mu+1)=v\right\},
$$

where $\mu \geq 1$ is an integer. Then, $\left|\mathcal{C}_{h, v}(\mu)\right|=\frac{1}{(\mu+1)^{2}} \cdot \frac{n}{\mathfrak{c}(n)}$, where $|\cdot|$ denotes the cardinality of a discrete set.

Lemma 1: Under any stationary and ergodic mobility model, at any time $t$, it holds that:

1) When $\mathfrak{c}(n)=O(\log n)$, define the number of cells in $\mathcal{C}_{h, v}(\mu)$ containing at least 2 nodes as a random variable $\xi$, then there is a constant $\theta_{1}>0$ such that $\xi \geq \theta_{1} \cdot \frac{n}{\mathfrak{c}(n)}$, w.h.p. 
2) When $\mathfrak{c}(n)=\Omega(\log n)$, for all cells in the lattice $\mathbb{L}(n, \mathfrak{c}(n))$, the number of ad hoc nodes is uniform of order $\Theta(\mathfrak{c}(n))$ w.h.p..

Proof: For the first case, it can be easily proven by Chebychev's inequality (Lemma 6). For the second case, it can be proven by VC Theorem ( [30]) by a similar method to that of Lemma 18 in [31]. All the proofs are based on the fact that under any stationary and ergodic mobility model, the distribution of nodes at all times remains to be uniform.

Next, we give an important result of the spatial multiplexing in extended MANETs.

Lemma 2: Under a Gaussian channel model $\mathscr{C}_{\text {gau }}(\alpha)$ with $\alpha>2$, when $\mathfrak{l}_{\mathbf{S}}=\Omega(1)$, there exists a strategy $\mathbf{S}$ under which there is a scheduling set $\mathcal{S}^{t}$ such that

1) $\left|\mathcal{S}^{t}\right| \geq \kappa_{0} \cdot n$, where $\left|\mathcal{S}^{t}\right|$ represents the total number of links contained in $\mathcal{S}^{t}$, and $\kappa_{0}>0$ is a constant;

2) for any link $i^{t} \in \mathcal{S}^{t}, R_{i}^{t}=\Omega\left(\left(\mathfrak{l}_{\mathbf{S}}\right)^{-\alpha}\right)$.

Proof: We will prove the lemma according to $\mathfrak{l}_{\mathbf{S}}$.

(a) When $\mathfrak{l}_{\mathbf{S}}=\Theta(1)$, i.e., $\mathfrak{c}(n)=\Theta(1)$.

At a static slot $t$, for any $\mathcal{C}_{h, v}(\mu)$, according to Case 1 of Lemma 1 , there are at least $\theta_{1} \cdot \frac{n}{\mathfrak{c}(n)}$ cells containing 2 nodes. We limit the links within the same cells, and choose a link from each cell to construct the set $\mathcal{S}^{t}$. Obviously, it holds that $\left|\mathcal{S}^{t}\right| \geq \theta_{1} \cdot n / \mathfrak{c}(n)$.

For any link $i^{t} \in \mathcal{S}^{t}$, the strength of signal at the receiver $\mathbf{r}_{i}^{t}$ is bounded by

$$
\mathrm{S}_{i}^{t} \geq P \cdot \min \left\{1,(\sqrt{2 \cdot \mathfrak{c}(n)})^{-\alpha}\right\}=\Theta(1) .
$$

The sum of interference at $\mathbf{r}_{i}^{t}$ is bounded as

$$
\mathrm{I}_{i}^{t} \leq 8 P \cdot(\mathfrak{c}(n))^{-\frac{\alpha}{2}} \cdot \sum_{k=0}^{\infty} \frac{1}{((\mu+1) k+\mu)^{\alpha}}
$$

Since $\alpha>2$, then $\mathrm{I}_{i}^{t}=O(1)$. According to Equation (1), it holds that $R_{i}^{t}=\Theta(1)$. Since $\mathfrak{c}(n)=\Theta(1)$, we get that $R_{i}^{t}=\Omega\left((\mathfrak{c}(n))^{-\frac{\alpha}{2}}\right)$.

(b) When $\mathfrak{l}_{\mathbf{S}}=\Omega(\sqrt{\log n})$, i.e., $\mathfrak{c}(n)=\Omega(\log n)$.

According to Case 2 of Lemma 1, there is a constant $\theta_{3}>0$ such that $n_{i, j} \geq \theta_{3} \cdot \mathfrak{c}(n)$ for all cells in $\mathbb{L}(n, \mathfrak{c}(n))$. Next, we construct the set $\mathcal{S}^{t}$. From any cell $C_{i, j} \in \mathcal{C}_{h, v}(3)$, choose $\theta_{3} \cdot \mathfrak{c}(n)$ nodes, and correspondingly choose $\theta_{3} \cdot \mathfrak{c}(n)$ nodes from the cell $C_{i, j+2}$ (or $C_{i+2, j}$ ). We make these $\theta_{3} \cdot \mathfrak{c}(n)$ pairs communicate directly, and finally obtain the set $\mathcal{S}^{t}$ consisting of $\left|\mathcal{C}_{h, v}(3)\right| \cdot 2 \theta_{3} \cdot \mathfrak{c}(n)$ links. Then, it holds that $\left|\mathcal{S}^{t}\right| \geq \theta_{2} \cdot n$, where $\theta_{2} \leq \frac{1}{8} \cdot \theta_{3}$.

For any link $i^{t} \in \mathcal{S}^{t}$, the strength of signal at $\mathbf{r}_{i}^{t}$ is:

$$
\mathrm{S}_{i}^{t} \geq P \cdot(\sqrt{10 \cdot \mathfrak{c}(n)})^{-\alpha}=\Omega\left((\mathfrak{c}(n))^{-\frac{\alpha}{2}}\right) .
$$

The sum of interference at $\mathbf{r}_{i}^{t}$ is bounded by

$$
\begin{aligned}
\mathrm{I}_{i}^{t} \leq & P \cdot\left(\theta_{3} \mathfrak{c}(n)-1\right) \cdot(\mathfrak{c}(n))^{-\frac{\alpha}{2}} \\
& +8 P \cdot \theta_{3} \mathfrak{c}(n) \cdot(\mathfrak{c}(n))^{-\frac{\alpha}{2}} \sum_{k=0}^{\infty} \frac{1}{(4 k+1)^{\alpha}} \\
\leq & P \cdot\left(1+8 \theta_{4}\right) \cdot(\mathfrak{c}(n))^{1-\frac{\alpha}{2}},
\end{aligned}
$$

where $\theta_{4}>\sum_{k=0}^{\infty} \frac{1}{(4 k+1)^{\alpha}}$ is a constant with $\alpha>2$, then

$$
\mathrm{I}_{i}^{t}=O\left((\mathfrak{c}(n))^{1-\frac{\alpha}{2}}\right),
$$

and then, $\mathrm{I}_{i}^{t}=o(1)$. According to Equation (1), it holds that

$$
R_{i}^{t}=\Omega\left((\mathfrak{c}(n))^{-\frac{\alpha}{2}}\right) .
$$

(c) When $\mathfrak{l}_{\mathbf{S}}=\Omega(1)$ and $\mathfrak{l}_{\mathbf{S}}=O(\sqrt{\log n})$, i.e., $\mathfrak{c}(n)=\Omega(1)$ and $\mathfrak{c}(n)=O(\log n)$.

By using Case 1 of Lemma 1 and a similar procedure of the case when $\mathfrak{l}_{\mathbf{S}}=\Omega(\sqrt{\log n})$, we can prove the result of this case.

From Lemma 2, the following result obviously holds.

Lemma 3: During any static slot $t$, the total throughput of network can be, w.h.p., of order $\Omega\left(n \cdot\left(\mathfrak{l}_{\mathbf{s}}\right)^{-\alpha}\right)$ under the communication strategy with $\mathfrak{l}_{\mathbf{s}}$.

2) Duration of Contact Intervals: Now, we derive the duration of contact intervals under a given communication strategy $\mathbf{S}$, denoted by $\tau_{C}\left(\mathfrak{l}_{\mathbf{S}}\right)$.

Lemma 4: Under a communication strategy $\mathbf{S}$, w.h.p., $\mathbf{E}\left(\tau_{C}\left(\mathfrak{l}_{\mathbf{S}}\right)\right)$ is of order $^{3}$

$$
\left\{\begin{array}{lll}
\Omega\left(\frac{\left(\mathfrak{l}_{\mathbf{S}}\right)^{2}}{\log n \cdot n^{\gamma}}\right) & \text { when } & \mathfrak{l}_{\mathbf{S}}:\left[n^{\frac{\gamma}{2}} \cdot \sqrt{\log n}, \sqrt{n}\right] \\
\Omega(1) & \text { when } & \mathfrak{l}_{\mathbf{S}}:\left[1, n^{\frac{\gamma}{2}} \cdot \sqrt{\log n}\right]
\end{array}\right.
$$

Proof: By Lemma 9, we study directly the first exit time to derive the order of contact time.

(a) When $\mathfrak{l}_{\mathbf{S}}=\Omega\left(n^{\frac{\gamma}{2}} \cdot \sqrt{\log n}\right)$.

Let $\left(x_{t}, y_{t}\right)$ denote the cell containing the node $i^{t}$, and define

$$
\begin{aligned}
\tau_{E}^{\mathrm{h}, x}(h) & :=\inf \left\{t \geq 0:\left|x_{t}-x_{0}\right| \geq h\right\} ; \\
\tau_{E}^{\mathrm{h}, y}(v) & :=\inf \left\{t \geq 0:\left|y_{t}-y_{0}\right| \geq v\right\} .
\end{aligned}
$$

By a simple geometrical argument, we have

$$
\mathbf{P}\left(\tau_{E}\left(\mathfrak{l}_{\mathbf{S}}\right) \leq L\right) \leq \mathbf{P}\left(\tau_{E}^{\mathrm{h}, x}\left(\frac{\mathfrak{l}_{\mathbf{S}}}{2 n^{\frac{\gamma}{2}}}\right) \leq L \vee \tau_{E}^{\mathrm{h}, y}\left(\frac{\mathfrak{l}_{\mathbf{S}}}{2 n^{\frac{\gamma}{2}}}\right) \leq L\right) .
$$

Using the union bound, we can obtain that

$$
\mathbf{P}\left(\tau_{E}\left(\mathfrak{l}_{\mathbf{S}}\right) \leq L\right) \leq 2 \mathbf{P}\left(\tau_{E}^{\mathrm{h}, x}\left(\frac{\mathfrak{l}_{\mathbf{S}}}{2 n^{\frac{\gamma}{2}}}\right) \leq L\right) .
$$

Before the phase $\tau_{E}\left(\mathfrak{l}_{\mathbf{S}}\right)$, it holds that

$$
x_{t}=x_{0}+\sum_{i=0}^{t} s_{i}
$$

where all $s_{i}$ are i.i.d. random variables taking their values in $\{-1,0,1\}$ with probabilities $\left\{\frac{1}{4}, \frac{1}{2}, \frac{1}{4}\right\}$, respectively. Due to the symmetry of $x_{t}$, it is clear that the reflection principle for 1-D random walk also holds in case of $x_{t}$. Thus, we have

$$
\mathbf{P}\left(\tau_{E}^{\mathrm{h}, x}\left(\frac{\mathfrak{l}_{\mathbf{S}}}{2 n^{\frac{\gamma}{2}}}\right) \leq L\right) \leq 2 \cdot \mathbf{P}\left(x_{\lfloor L\rfloor}-x_{0} \geq \frac{\mathfrak{l}_{\mathbf{S}}}{2 n^{\frac{\gamma}{2}}}\right),
$$

where $\lfloor\cdot\rfloor$ denotes the greatest integer function. Each $s_{i}$ has mean 0 and variance $\frac{1}{2}$, then

$$
\operatorname{Var}\left(x_{t}-x_{0}\right)=t / 2 \text {. }
$$

By using Lemma 7, we have, for $t \geq \frac{l_{\mathrm{s}}}{2 n^{\frac{\gamma}{2}}}$,

$\mathbf{P}\left(x_{t}-x_{0} \geq \frac{\mathfrak{l}_{\mathbf{S}}}{2 n^{\frac{\gamma}{2}}}\right)=\mathbf{P}\left(x_{t}-x_{0} \geq \sqrt{\frac{t}{2}} \cdot \frac{\mathfrak{l}_{\mathbf{S}}}{\sqrt{2 t} \cdot n^{\frac{\gamma}{2}}}\right)$

${ }^{3}$ Throughout the paper, for a regime $\mathfrak{l}_{\mathbf{S}}:[f(n), g(n)]$ in an expression, if $f(n)=\Theta(g(n))$, then $\mathfrak{l}_{\mathbf{S}}=\Theta(f(n))$; if $f(n)=\omega(g(n))$, it means that the regime $\mathfrak{l}_{\mathbf{S}}:[f(n), g(n)]$ is empty. 


$$
\leq \exp \left(-\left(\mathfrak{l}_{\mathbf{s}} / n^{\frac{\gamma}{2}}\right)^{2} \cdot \frac{1}{8 t}\right)
$$

Let $L=\frac{\left(\mathfrak{l}_{\mathbf{S}}\right)^{2}}{16 \log n \cdot n^{\gamma}}$, we have

$$
\mathbf{P}\left(\tau_{E}\left(\mathfrak{l}_{\mathbf{S}}\right) \leq \frac{\left(\mathfrak{l}_{\mathbf{S}}\right)^{2}}{16 \log n \cdot n^{\gamma}}\right) \leq \frac{4}{n^{2}}
$$

Then, $\mathbf{E}\left(\tau_{E}\left(\mathfrak{l}_{\mathbf{S}}\right)\right)=\Omega\left(\frac{\left(\mathfrak{l}_{\mathbf{S}}\right)^{2}}{\log n \cdot n^{\gamma}}\right)$.

(b) When $\mathfrak{l}_{\mathbf{S}}=O\left(n^{\frac{\gamma}{2}} \cdot \sqrt{\log n}\right)$.

It is straightforward that $\tau_{E}\left(\mathfrak{l}_{\mathbf{s}}\right) \geq L_{\mathrm{p}}$. Since $L_{\mathrm{p}}=\Theta(1)$, we get that $\mathbf{E}\left(\tau_{E}\left(\mathfrak{l}_{\mathbf{S}}\right)\right)=\Omega(1)$.

3) Duration of Waiting Intervals: Let $\tau_{W}\left(\mathfrak{l}_{\mathbf{S}}\right)$ denote the duration of waiting intervals in a given communication strategy S. Then, we have

Lemma 5: Under a communication strategy $\mathbf{S}$, w.h.p., $\mathbf{E}\left(\tau_{W}\left(\mathfrak{l}_{\mathbf{S}}\right)\right)$ is of order

$$
\left\{\begin{array}{lll}
\Omega\left(\frac{n^{1-\gamma}}{\log n}\right) & \text { when } & \mathfrak{l}_{\mathbf{S}}:[1, \sqrt{n}) \\
\Theta\left(\frac{n}{\left(\mathfrak{l}_{\mathbf{S}}\right)^{2}}+\frac{\log n}{n^{\gamma-1}}\right) & \text { when } & \mathfrak{l}_{\mathbf{S}}:[1, \sqrt{n}) \cap\left[1, n^{\frac{\gamma}{2}}\right] \\
\Theta(1) & \text { when } & \mathfrak{l}_{\mathbf{S}}=\Theta(\sqrt{n})
\end{array}\right.
$$

Proof: Let $\tau_{H}$ denote the time required by nodes to enter the same cell, starting from their initial random and uniformly distributed positions, i.e., the first hitting time (Definition 2); and let $\tau_{R}$ denote the time between successive hitting states, i.e., the return time (Definition 2). Now, we analyze the order of $\tau_{W}\left(l_{\mathbf{S}}\right)$, i.e., the duration of waiting intervals in a given twohop communication strategy $\mathbf{S}$ under the hybrid random walk mobility model, according to $\mathbb{L}\left(n,\left(\mathfrak{l}_{\mathbf{S}}\right)^{2}\right)$. In the following analysis, $\varphi_{i}, i \geq 1$, denote some proper constants, the specific values of them do not change our results in order sense.

Recall that the mobility model is based on a lattice (torus) $\mathbb{L}\left(n, n^{\gamma}\right)$. According to Corollary 1, w.h.p., it holds that

$$
\mathbf{E}\left(\tau_{H}\right)=\Theta\left(n^{1-\gamma} \cdot \log n\right) ; \mathbf{E}\left(\tau_{R}\right)=\Theta\left(n^{1-\gamma}\right)
$$

(a) When $\mathfrak{l}_{\mathbf{S}}:[1, \sqrt{n}) \cap\left[1, n^{\frac{\gamma}{2}}\right]$. We have

$$
\tau_{W}\left(\mathfrak{l}_{\mathbf{S}}\right)=\sum_{k=0}^{\infty}\left(\tau_{H}+k \cdot \tau_{R}\right) \cdot\left(1-\varphi_{2} \frac{\left(\mathfrak{l}_{\mathbf{S}}\right)^{2}}{n^{\gamma}}\right)^{k} \cdot \varphi_{1} \cdot \frac{\left(\mathfrak{l}_{\mathbf{S}}\right)^{2}}{n^{\gamma}},
$$

where $0^{0}=1$. Then,

$$
\begin{aligned}
& \mathbf{E}\left(\tau_{W}\left(\mathfrak{l}_{\mathbf{S}}\right)\right) \\
& =\frac{\varphi_{1}\left(\mathfrak{l}_{\mathbf{S}}\right)^{2}}{n^{\gamma}} \cdot\left(\mathbf{E}\left(\tau_{H}\right) \cdot \frac{n^{\gamma}}{\left(\mathfrak{l}_{\mathbf{s}}\right)^{2}}+\mathbf{E}\left(\tau_{R}\right) \cdot \frac{n^{4 \frac{\gamma}{2}}-\left(\mathfrak{l}_{\mathbf{S}}\right)^{2} \cdot n^{\gamma}+\left(\mathfrak{l}_{\mathbf{S}}\right)^{4}}{\left(\mathfrak{l}_{\mathbf{S}}\right)^{4}}\right) \\
& =\Theta\left(\mathbf{E}\left(\tau_{H}\right)\right)+\Theta\left(\mathbf{E}\left(\tau_{R}\right) \cdot \frac{n^{\gamma}}{\left(\mathfrak{l}_{\mathbf{s}}\right)^{2}}\right)
\end{aligned}
$$

Hence, we have that

$$
\mathbf{E}\left(\tau_{W}\left(\mathfrak{l}_{\mathbf{S}}\right)\right)=\Theta\left(\frac{n}{\left(\mathfrak{l}_{\mathbf{S}}\right)^{2}}+n^{1-\gamma} \cdot \log n\right) .
$$

(b) When $\mathfrak{l}_{\mathbf{S}}=\Theta(\sqrt{n})$. It is straightforward that

$$
\mathbf{E}\left(\tau_{W}\left(\mathfrak{l}_{\mathbf{S}}\right)\right)=\Theta(1) .
$$

(c) A general lower bound of $\mathbf{E}\left(\tau_{W}\left(\mathfrak{l}_{\mathbf{S}}\right)\right)$ for $\mathfrak{l}_{\mathbf{S}}:[1, \sqrt{n})$. By geometrical argument, it is clear that

$$
\mathbf{E}\left(\tau_{W}\left(\mathfrak{l}_{\mathbf{S}}\right)\right)=\Theta\left(\mathbf{E}\left(\tau_{W}\left(\varphi_{3} \sqrt{n}-\mathfrak{l}_{\mathbf{S}}\right)\right)\right) .
$$

Then, according to Lemma 4 , we have that for $\mathfrak{l}_{\mathbf{S}}:[1, \sqrt{n})$,

$$
\mathbf{E}\left(\tau_{W}\left(\mathfrak{l}_{\mathbf{S}}\right)\right)=\Omega\left(n^{1-\gamma} / \log n\right) .
$$

Combining all cases above, we complete the proof.

\section{Capacity and Delay Analysis}

Following the setting, we set the number of sessions be of $\Theta(n)$. Since we only care the order of results, we will directly introduce some constant coefficients in the proofs of Theorem 1 without detailed explanation. Specifically, all used $c_{i}, v_{i}$, and $\tau_{i}$, for $i \geq 1$, are some proper positive constants.

We propose the following result.

Theorem 1: Under a Gaussian channel model $\mathscr{C}_{\text {gau }}(\alpha)$, by using the two-hop strategy $\mathbf{S}$ with the critical distance $\mathfrak{l}_{\mathbf{S}}$, the average throughput $\lambda(\mathbf{S}, n)$, w.h.p., can be achieved of:

$$
\left\{\begin{array}{lll}
\Omega\left(\frac{\log n \cdot n^{\gamma}}{\left(l_{\mathbf{S}}\right)^{2}}\right) & \text { when } & \mathfrak{l}_{\mathbf{S}}:\left[n^{\frac{\gamma}{2}} \sqrt{\log n}, \sqrt{n}\right] \\
\Theta(1) & \text { when } & \mathfrak{l}_{\mathbf{S}}:\left[1, n^{\frac{\gamma}{2}} \sqrt{\log n}\right] \cap[1, \sqrt{n}]
\end{array}\right.
$$

The average delay, denoted by $\mathbf{E}(D(\mathbf{S}, n))$, is of

$$
\left\{\begin{array}{lll}
\Theta\left(\frac{n}{\left(I_{\mathbf{S}}^{2}\right.}+\frac{\log n}{n^{\gamma-1}}\right) & \text { when } & \mathfrak{l}_{\mathbf{S}}:\left[1, n^{\frac{\gamma}{2}}\right] \cap[1, \sqrt{n}) \\
\Omega\left(\frac{n^{-1}}{\log n}+1\right) & \text { when } & \mathfrak{l}_{\mathbf{S}}:\left[n^{\frac{\gamma}{2}}, \sqrt{n}\right) \\
O\left(\frac{n^{-1} \gamma}{\log n}+1\right) & \text { when } & \mathfrak{l}_{\mathbf{S}}=\Theta(\sqrt{n})
\end{array}\right.
$$

\section{A. Capacity Analysis}

By Lemma 4, when $c_{1} \cdot n^{\frac{\gamma}{2}} \cdot \sqrt{\log n} \leq \mathfrak{l}_{\mathbf{S}} \leq c_{2} \sqrt{n}$, the duration of $\mathrm{S} \rightarrow \mathrm{R}$ phase and $\mathrm{R} \rightarrow \mathrm{D}$ phase can be denoted by $\tau_{1} \cdot \frac{\left(I_{\mathbf{S}}\right)^{2}}{\log n \cdot n^{\gamma}}$; when $c_{3} \leq \mathfrak{l}_{\mathbf{S}} \leq c_{1} \cdot n^{\frac{\gamma}{2}} \cdot \sqrt{\log n}$, the duration of $\mathrm{S} \rightarrow \mathrm{R}$ phase and $\mathrm{R} \rightarrow \mathrm{D}$ phase can be denoted by a constant $\tau_{1} \cdot\left(c_{1}\right)^{2}$.

1) When $c_{1} \cdot n^{\frac{\gamma}{2}} \cdot \sqrt{\log n} \leq \mathfrak{l}_{\mathbf{S}} \leq c_{2} \sqrt{n}$ : Consider any communication-pairs under the scheme $\mathbf{S}$, denote the distance between the pairs by $\mathfrak{d}^{t} \in\left(0, \mathfrak{l}_{\mathbf{S}}\right]$. We compute the achievable traffic between the pairs in two disjointed cases, i.e., $\mathfrak{d}^{t} \in$ $\left[c_{1} \cdot n^{\frac{\gamma}{2}} \cdot \sqrt{\log n}, \mathfrak{l}_{\mathbf{S}}\right]$ and $\mathfrak{d}^{t} \in\left(0, c_{1} \cdot n^{\frac{\gamma}{2}} \cdot \sqrt{\log n}\right)$. Then, divide the total achievable traffic of both cases by the duration of $\mathrm{S} \rightarrow \mathrm{R}$ phase and $\mathrm{R} \rightarrow \mathrm{D}$ phase, i.e., $\tau_{1} \cdot \frac{\left(\mathrm{I}_{\mathbf{S}}\right)^{2}}{\log n \cdot n^{\gamma}}$, we can obtain the average throughput under the scheme $\mathbf{S}$. For the latter case, the duration is not less than $\tau_{1} \cdot\left(c_{1}\right)^{2}$ that is feasible even when $\mathfrak{l}_{\mathbf{S}}=c_{3}<c_{1} \cdot n^{\frac{\gamma}{2}} \cdot \sqrt{\log n}$. Therefore, according to Lemma 3 , there exists a constant $v_{1}>0$ such that the average throughput is bounded as in Equation (6 8). Since $\alpha>2$, there exists a constant $c_{7}<c_{5}$ such that $\lambda(\mathbf{S}, n) \geq c_{7} \cdot \log n \cdot n^{\gamma} /\left(\mathfrak{l}_{\mathbf{S}}\right)^{2}$. Then, we have that $\lambda(\mathbf{S}, n)=\Omega\left(\frac{\log n \cdot n^{\gamma}}{\left(\mathbf{S}_{\mathbf{S}}\right)^{2}}\right)$.

2) When $c_{3} \leq \mathfrak{l}_{\mathbf{S}} \leq c_{1} \cdot n^{\frac{\gamma}{2}} \cdot \sqrt{\log n}$ : For this case, there exists a constant $v_{2}$ such that

$$
\lambda(\mathbf{S}, n) \geq v_{2} \cdot \tau_{2} \cdot\left(c_{3}\right)^{-\alpha} / \tau_{1}\left(c_{1}\right)^{2} .
$$

Thus, $\lambda(\mathbf{S}, n)=\Omega(1)$. Under any noncooperative communication strategy $\mathbf{S}$, due to the presence of interference, the average throughput holds that $\lambda(\mathbf{S}, n)=O(1)$. Then, we get that $\lambda(\mathbf{S}, n)=\Theta(1)$.

\section{B. Delay Analysis}

For simplicity, let $\mathbf{E}(D)$ denote $\mathbf{E}(D(\mathbf{S}, n))$ in this part. According to the definition of network delay, and based on Lemma 5 , we can easily obtain the results.

1) When $\mathfrak{l}_{\mathbf{S}}:\left[1, n^{\frac{\gamma}{2}}\right] \cap[1, \sqrt{n})$ : It holds that

$$
\mathbf{E}(D)=\Theta\left(\frac{n}{\left(\mathfrak{l}_{\mathbf{S}}\right)^{2}}+n^{1-\gamma} \cdot \log n+2\right) .
$$




$$
\begin{aligned}
\lambda(\mathbf{S}, n) & \geq \frac{1}{\frac{\tau_{1}\left(\mathfrak{l}_{\mathbf{S}}\right)^{2}}{\log n \cdot n^{\gamma}}} \cdot\left(\int_{c_{1} \cdot n^{\frac{\gamma}{2}} \cdot \sqrt{\log n}}^{\mathfrak{l}_{\mathbf{S}}} v_{1} \cdot x^{-\alpha} d \frac{\tau_{1} \cdot x^{2}}{\log n \cdot n^{\gamma}}+\tau_{2} \cdot v_{1} \cdot\left(c_{3}\right)^{-\alpha}\right) \\
& =\frac{1}{(\alpha-2) \cdot\left(\mathfrak{l}_{\mathbf{S}}\right)^{2}} \times\left(\frac{1}{\left(c_{1}\right)^{\alpha-2} \cdot n^{(\alpha-2) \frac{\gamma}{2}} \cdot(\log n)^{\frac{\alpha}{2}-1}}-\frac{1}{\left(\mathfrak{l}_{\mathbf{S}}\right)^{\alpha-2}}\right)+\frac{v_{1} \cdot \tau_{2} \cdot\left(c_{3}\right)^{-\alpha}}{\tau_{1}} \cdot \frac{\log n \cdot n^{\gamma}}{\left(\mathfrak{l}_{\mathbf{S}}\right)^{2}} \\
& =c_{4} \cdot \frac{1}{n^{(\alpha-2) \frac{\gamma}{2}} \cdot(\log n)^{\frac{\alpha}{2}-1} \cdot\left(\mathfrak{l}_{\mathbf{S}}\right)^{2}}+c_{5} \cdot \frac{\log n \cdot n^{\gamma}}{\left(\mathfrak{l}_{\mathbf{S}}\right)^{2}}-c_{6} \cdot \frac{1}{\left(\mathfrak{l}_{\mathbf{S}}\right)^{\alpha}} .
\end{aligned}
$$

Here, $\tau_{2} \leq \tau_{1} \cdot\left(c_{1}\right)^{2}, c_{4}=\frac{2 v_{1}}{(\alpha-2) \cdot\left(c_{1}\right)^{\alpha-2}}, c_{5}=\frac{v_{1} \cdot \tau_{2} \cdot\left(c_{3}\right)^{-\alpha}}{\tau_{1}}$, and $c_{6}=\frac{2 v_{1}}{\alpha-2}$.

2) When $\mathfrak{l}_{\mathbf{S}}:\left[n^{\frac{\gamma}{2}}, n^{\frac{\gamma}{2}} \cdot \sqrt{\log n}\right] \cap[1, \sqrt{n})$ : It holds that

$$
\mathbf{E}(D)=\Omega\left(\frac{n^{1-\gamma}}{\log n}+2\right) \text {. }
$$

3) When $\mathfrak{l}_{\mathbf{S}}:\left[n^{\frac{\gamma}{2}} \cdot \sqrt{\log n}, \sqrt{n}\right)$ : It holds that

$$
\mathbf{E}(D)=\Omega\left(\frac{n^{1-\gamma}}{\log n}\right)+O\left(\frac{\left(\mathfrak{l}_{\mathbf{s}}\right)^{2}}{\log n \cdot n^{\gamma}}\right)+\Theta(2) .
$$

Then, $\mathbf{E}(D)=\Omega\left(\frac{n^{1-\gamma}}{\log n}+1\right)$.

4) When $\mathfrak{l}_{\mathbf{S}}=\Theta(\sqrt{n})$ : It holds that

$$
\mathbf{E}(D)=\Theta(1)+O\left(\frac{n}{\log n \cdot n^{\gamma}}\right)+\Theta(2) .
$$

Then, $\mathbf{E}(D)=O\left(\frac{n^{1-\gamma}}{\log n}+1\right)$.

By dividing the regime of freedom degree $\gamma:[0,1]$ into three parts, i.e., $\gamma=0,0<\gamma<1$, and $\gamma=1$, we can obtain the final results described in Fig.1, Fig.2, and Fig.3.

\section{CONCLUSION}

We study the asymptotic unicast capacity and delay for mobile ad hoc networks under the Gaussian channel model, rather than the protocol model and physical model as in the literature. We derive the capacity and delay depending on different freedom degree of nodes, and deduce some useful results.

There are two interesting issues left to study. The first is to examine the asymptotic capacity and delay under communication strategies with redundancy. The second is to extend the results to other communication patterns, such as multicast and broadcast sessions.

\section{ACKNOWLEDGMENTS}

The research of authors is partially supported by the National Science Foundation for Post-doctoral Scientists of China under grant No. 2012M510118, the National Basic Research Program of China (973 Program) under grants No. 2010CB328101 and 2011CB302804, the Program for Changjiang Scholars and Innovative Research Team in University, the Shanghai Key Basic Research Project under grant No. 10DJ1400300, the NSF CNS-1035894, the Program for Zhejiang Provincial Key Innovative Research Team, and the Program for Zhejiang Provincial Overseas High-Level Talents (One-hundred Talents Program).

\section{REFERENCES}

[1] M. Grossglauser and D. Tse, "Mobility increases the capacity of ad hoc wireless networks," IEEE/ACM Trans. on Networking, vol. 10, no. 4, pp. 477-486, 2002.

[2] P. Gupta and P. R. Kumar, "The capacity of wireless networks," IEEE Trans. on Information Theory, vol. 46, no. 2, pp. 388-404, 2000.

[3] M. Franceschetti, O. Dousse, D. Tse, and P. Thiran, "Closing the gap in the capacity of wireless networks via percolation theory," IEEE Trans. on Information Theory, vol. 53, no. 3, pp. 1009-1018, 2007.

[4] C. Hu, X. Wang, and F. Wu, "Motioncast: On the capacity and delay tradeoffs," in Proc. ACM Mobihoc 2009.

[5] M. Neely and E. Modiano, "Capacity and delay tradeoffs for ad hoc mobile networks," IEEE Trans. on Information Theory, vol. 51, no. 6, pp. 1917-1937, 2005.

[6] S. Toumpis and A. J. Goldsmith, "Large wireless networks under fading, mobility, and delay constraints," in Proc. IEEE INFOCOM 2004.

[7] Z. Wang and H. Sadjadpour, "Capacity-delay tradeoff for information dissemination modalities in wireless networks," in Proc. IEEE ISIT 2008.

[8] A. E. Gamal, J. Mammen, B. Prabhakar, and D. Shah, "Throughputdelay trade-off in wireless networks," in Proc. IEEE INFOCOM 2004.

[9] G. Sharma and R. Mazumdar, "Scaling laws for capacity and delay in wireless ad hoc networks with random mobility," in Proc. IEEE ICC 2004.

[10] X. Lin, G. Sharma, R. Mazumdar, and N. Shroff, "Degenerate delay-capacity tradeoffs in ad-hoc networks with brownian mobility," IEEE/ACM Trans. on Networking, vol. 14, no. SI, p. 2784, 2006.

[11] G. Sharma and R. Mazumdar, "Delay and capacity trade-off in wireless ad hoc networks with random way-point mobility," Dept. Elect. Comput. Eng., Purdue Univ.,West Lafayette, IN, Available: http://ece.purdue.edu/ gsharma/, Tech. Rep., 2005.

[12] G. Sharma, R. Mazumdar, and N. Shroff, "Delay and capacity trade-offs in mobile ad hoc networks: A global perspective," IEEE/ACM Trans. on Networking, vol. 15, no. 5, pp. 981-992, 2007.

[13] L. Ying, S. Yang, and R. Srikant, "Optimal delay-throughput tradeoffs in mobile ad hoc networks," IEEE Trans. on Information Theory, vol. 54, no. 9, pp. 4119-4143, 2008.

[14] _ "Coding achieves the optimal delay-throughput tradeoffs in mobile ad-hoc networks," in Proc. IEEE WiOpt 2007.

[15] M. Garetto, P. Giaccone, and E. Leonardi, "Capacity scaling in delay tolerant networks with heterogeneous mobile nodes," in Proc. ACM MobiHoc 2007.

[16] W. Huang, X. Wang, and Q. Zhang, "Capacity scaling in mobile wireless ad hoc network with infrastructure support," in Proc. IEEE ICDCS 2010.

[17] M. Garetto and E. Leonardi, "Restricted Mobility Improves DelayThroughput Tradeoffs in Mobile Ad Hoc Networks," IEEE Trans. on Information Theory, vol. 56, no. 10, pp. 5016-5029, 2010.

[18] X. Wang, Y. Bei, Q. Peng, and L. Fu, "Speed improves delay-capacity tradeoff in MotionCast," IEEE Trans. on Parallel and Distributed Systems, vol. 22, no. 5, pp. $729-742,2011$.

[19] S. Zhou and L. Ying, "On delay constrained multicast capacity of largescale mobile ad-hoc networks," in Proc. IEEE INFOCOM 2010.

[20] G. Resta and P. Santi, "On the fundamental limits of broadcasting in wireless mobile networks," in Proc. IEEE INFOCOM 2010.

[21] P. Jacquet, B. Mans, and G. Rodolakis, "Information propagation speed in mobile and delay tolerant networks," IEEE Trans. on Information Theory, vol. 56, no. 10, pp. 5001-5015, 2010. 
[22] J. Jose, A. Abdel-Hadi, P. Gupta, and S. Vishwanath, "On the impact of mobility on multicast capacity of wireless networks," in Proc. IEEE INFOCOM 2010.

[23] A. Keshavarz-Haddad and R. Riedi, "Multicast capacity of large homogeneous multihop wireless networks," in Proc. IEEE WiOpt 2008.

[24] S. Li, Y. Liu, and X.-Y. Li, "Capacity of large scale wireless networks under gaussian channel model," in Proc. ACM MobiCom 2008.

[25] Y. Wang, X. Chu, X. Wang, and Y. Cheng, "Optimal Multicast Capacity and Delay Tradeoffs in MANETs: A Global Perspective," in Proc. IEEE INFOCOM 2011.

[26] A. El Gamal, J. Mammen, B. Prabhakar, and D. Shah, "Optimal throughput-delay scaling in wireless networks-Part II: constant-size packets," IEEE Trans. on Information Theory, vol. 52, no. 11, pp. 51115116, 2006.

[27] C. Wang, X.-Y. Li, S. Tang, and C. Jiang, "Fundamental limits in mobile ad hoc networks under adaptive-rate communication model," Tech. Rep., http://www.cs.iit.edu/\%7Exli/paper/Submitted/Transactions-Manet.pdf.

[28] A. ÖzgÜr, O. LÉvÊque, and D. Tse, "Hierarchical Cooperation Achieves Optimal Capacity Scaling in Ad Hoc Networks," IEEE Trans. on Information Theory, vol. 53, no. 10, pp. 3549-3572, 2007.

[29] X. Wang, W. Huang, S. Wang, J. Zhang, and C. Hu, "Delay and capacity tradeoff analysis for motioncast," IEEE/ACM Trans. on Networking, vol. 19 , no. 5, pp. 1354-1367, oct. 2011.

[30] V. Vapnik and A. Chervonenkis, "On the uniform convergence of relative frequencies of events to their probabilities," Theory of Probability and its Applications, vol. 16, no. 2, pp. 264-280, 1971.

[31] X.-Y. Li, "Multicast capacity of wireless ad hoc networks," IEEE/ACM Trans. on Networking, vol. 17, no. 3, pp. 950-961, 2009.

[32] M. Mitzenmacher and E. Upfal, Probability and Computing: Randomized Algorithms and Probabilistic Analysis. Cambridge, U. K.: Cambridge Univ. Press, 2005.

[33] D. Aldous and J. Fill, Reversible Markov chains and random walks on graphs, 2002, Available: http://www.stat.berkeley.edu/\% 7Ealdous/RWG/book.html.

[34] J. Gross and J. Yellen, Graph theory and its applications. CRC press, 2006.

\section{APPENDIX A \\ PROBABILITY TOOLS}

Let $\mathbf{E}(X)$ and $\operatorname{Var}(X)$ denote the mean and variance of the random variable $X$, respectively. First, we recall two probability inequalities that are used in the analysis.

Lemma 6 (Chebyshev's Inequality, [32]): Let $X$ be a random variable, then

$$
\mathbf{P}(|X-\mathbf{E}(X)| \geq \epsilon) \leq \operatorname{Var}(X) / \epsilon^{2},
$$

where $\epsilon$ is an arbitrary positive value.

Lemma 7 (Hoeffding's Inequality, [32]): Let $X_{1}, X_{2}, \cdots$, $X_{n}$ be i.i.d. random variables taking values in $[-d, d]$ for $0<$ $d<\infty$, and suppose $\mathbf{E}\left(X_{i}\right)=0$ for all $i$. Let $S_{n}=\sum_{i=1}^{n} X_{i}$. Then, for all $\nu \in\left[0,2 \sqrt{\operatorname{Var}\left(S_{n}\right)} / d\right]$, it holds that

$$
\begin{gathered}
\mathbf{P}\left(S_{n} \geq \nu \cdot \sqrt{\operatorname{Var}\left(S_{n}\right)}\right) \leq \exp \left(-\nu^{2} / 4\right) . \\
\text { APPENDIX B } \\
\text { RANDOM WALKS ON GRAPHS }
\end{gathered}
$$

First, we introduce two key notions: first hitting time and first return time [33], which will be used for studying the delay under our scheme. As in [12], it is more convenient to define these two notions in discrete time. Let $X(t)$ be a Markov chain taking values in the set of states $\mathcal{S}_{X}$, and with a stationary distribution of $\mathfrak{X}$.

Definition 2 ([33]): The first hitting time for the set of states $\mathcal{A} \subseteq \mathcal{S}_{X}$, denoted by $\tau_{H}(\mathcal{A})$, is defined as

$$
\tau_{H}(\mathcal{A})=\inf \{t \geq 0: X(t) \in \mathcal{A}\}
$$

the first return time for $\mathcal{A} \subseteq \mathcal{S}_{X}$, denoted by $\tau_{R}(\mathcal{A})$, is defined as

$$
\tau_{R}(\mathcal{A})=\inf \{t \geq 1: X(t) \in \mathcal{A}, X(0) \in \mathcal{A}\},
$$

where $X(0)$ follows the distribution of $\mathfrak{X}$.

Next, we recall some results that will be used in the analysis of delay. We focus on a $d$-dimensional torus, denoted by $\mathbb{T}_{k}^{d}$, which is the set of $d$-dimensional integers $\mathbf{i}=\left(i_{1}, i_{2}, \cdots, i_{d}\right)$ modulo $k$, considered in the natural way as a $2 d$-regular graph, i.e., a graph where each vertex has $2 d$ neighbors [34], on $k^{d}$ vertices ( [33], Chapter 5, pp. 32-33.). We first introduce the result about these two notions for a single state in $\mathbb{T}_{k}^{d}$.

Lemma 8 ([33]): Denote the first hitting time and first return time for a single state on a $d$-dimensional torus $\mathbb{T}_{k}^{d}$ by $\tau_{H}$ and $\tau_{R}$, respectively, then, w.h.p., it holds that

$$
\mathbf{E}\left(\tau_{H}\right)=\Theta\left(k^{d} \log k^{d}\right), \mathbf{E}\left(\tau_{R}\right)=\Theta\left(k^{d}\right) .
$$

The description of mobility model is based on the 2dimensional torus lattices, say $\mathbb{L}(\mathfrak{a}, \mathfrak{c})$. A torus lattice $\mathbb{L}(\mathfrak{a}, \mathfrak{c})$ can be transferred into a 2-dimensional torus $\mathbb{T}_{k}^{d}$ with $d=2$ and $k=\sqrt{\frac{a}{c}}$. We also denote the first hitting time and first return time for a single state on a torus lattice $\mathbb{L}(\mathfrak{a}, \mathfrak{c})$ by $\tau_{H}$ and $\tau_{R}$, respectively. According to Lemma 8, we have,

Corollary 1: For a single state on a torus lattice $\mathbb{L}(\mathfrak{a}, \mathfrak{c})$, w.h.p., it holds that

$$
\mathbf{E}\left(\tau_{H}\right)=\Theta\left(\frac{\mathfrak{a}}{\mathfrak{c}} \cdot(\log \mathfrak{a}-\log \mathfrak{c})\right), \mathbf{E}\left(\tau_{H}\right)=\Theta\left(\frac{\mathfrak{a}}{\mathfrak{c}}\right) .
$$

Next, we introduce the notions of the first exit time [33]. Let $\mathcal{U}(x, r)$ denote the set of points within $\mathcal{R}(n)$ such that $|x-y| \leq r$.

Definition 3 ([33]): For any node $i$, the first exit time of $\mathcal{U}\left(i^{0}, r\right)$, denoted by $\tau_{E}(r)$, is defined as

$$
\tau_{E}(r)=\inf \left\{t \geq 0: i^{t} \notin \mathcal{U}\left(i^{0}, r\right)\right\} .
$$

Similar to the first exit time, we introduce the contact time.

Definition 4 ([11]): For any two nodes, say $i$ and $j$, the contact time $\tau_{C}(r)$, is defined as

$$
\tau_{C}(r)=\inf \left\{t>0: j^{t} \notin \mathcal{U}\left(i^{t}, r\right), j^{0} \in \mathcal{U}\left(i^{0}, r\right)\right\} .
$$

About the contact time and first exit time, by a simple geometrical argument, the following result holds.

Lemma 9 ( [33]): Under the hybrid random walk mobility model, w.h.p., it holds that $\mathbf{E}\left(\tau_{C}(r)\right)=\Theta\left(\mathbf{E}\left(\tau_{E}(r)\right)\right)$. 